\title{
On Edges Crossing Few Other Edges in Simple Topological Complete Graphs
}

\author{
Jan Kynčl and Pavel Valtr \\ Department of Applied Mathematics and Institute for Theoretical Computer Science \\ (ITI), Charles University, Malostranské nám. 25, 11800 Praha 1, Czech Republic
}

\begin{abstract}
We study the existence of edges having few crossings with the other edges in drawings of the complete graph (more precisely, in simple topological complete graphs). A topological graph $T=(V, E)$ is a graph drawn in the plane with vertices represented by distinct points and edges represented by Jordan curves connecting the corresponding pairs of points (vertices), passing through no other vertices, and having the property that any intersection point of two edges is either a common end-point or a point where the two edges properly cross. A topological graph is simple, if any two edges meet in at most one common point.

Let $h=h(n)$ be the smallest integer such that every simple topological complete graph on $n$ vertices contains an edge crossing at most $h$ other edges. We show that $\Omega\left(n^{3 / 2}\right) \leq h(n) \leq O\left(n^{2} / \log ^{1 / 4} n\right)$. We also show that the analogous function on other surfaces (torus, Klein bottle) grows as $c n^{2}$.
\end{abstract}

\section{Introduction}

A topological graph $T=(V, E)$ is a graph drawn in the plane with vertices represented by distinct points and edges represented by Jordan curves connecting the corresponding pairs of points (vertices), passing through no other vertices, and having the property that any intersection point of two edges is either a common end-point or a point where the two edges properly cross. A topological graph is simple, if any two edges meet in at most one common point.

One of the traditional themes in the area of graph drawings is to realize a given abstract graph as a topological graph so that the number of edge crossings is minimized. Here we consider a variant of a "dual" problem. We study realizations of the complete graph where each edge crosses "many" other edges.

Consider a network model drawn as a topological graph where the edge crossings are used for the exchange of some commodities (or information) between the two crossing edges. In any such model, edges with few crossings can exchange only small amounts of the commodities with the other edges within a time unit. This leads to the question about the existence of drawings in which each edge crosses "many" other edges.

If we can choose the underlying abstract graph on $n$ vertices, then we can realize it with each edge crossing $\Omega\left(n^{2}\right)$ other edges. E.g., take the vertices of a regular $n$-gon and connect each vertex by straight-line segments with the $\approx n / 3$ 
opposite vertices. Each edge in the obtained topological graph crosses at least $\approx n^{2} / 9$ other edges. Moreover, each edge is realized by a straight-line segment, thus it is a so-called rectilinear drawing (sometimes also called a geometric graph). If the underlying graph is fixed then the situation is much more complicated. In this paper we restrict our attention to topological complete graphs, i.e., to realizations of (abstract) complete graphs. We are not aware of any result for other classes of graphs.

If any two edges are allowed to cross each other at most twice, then there are various realizations of the complete graph with each edge crossing $\Omega\left(n^{2}\right)$ other edges. E.g., take $n$ points (vertices) on a short horizontal segment $s$ and for any two vertices $a, b$, connect $a$ and $b$ by an arc constructed as follows. Let $U(a, b)$ be the unit circle going through $a$ and $b$ and having the center above $s$. Then the edge $a b$ is drawn as the arc obtained from $U(a, b)$ by removing the part below the segment $a b$. Then any two edges with no common vertex cross once or twice. A different example of such a drawing is described in [14. In this paper we show that the situation is different for simple topological (complete) graphs.

According to the so-called crossing lemma [1, 9], if $T$ is a topological graph with $n$ vertices and $e \geq(3+\varepsilon) n$ edges then its crossing number is at least $\Omega\left(e^{3} / n^{2}\right)$, (i.e., it contains at least $\Omega\left(e^{3} / n^{2}\right)$ crossing pairs of edges). It follows that if $T$ is a topological complete graph then its crossing number is $\Omega\left(n^{4}\right)$ (this has also a quite easy direct proof). If $T$ is simple then there are at most $\left(\begin{array}{c}n-2 \\ 2\end{array}\right)=$ $O\left(n^{2}\right)$ crossings on each edge. It follows that a simple topological complete graph on $n$ vertices contains $\Omega\left(n^{2}\right)$ edges each of which crosses $\Omega\left(n^{2}\right)$ other edges.

We study the existence of edges with (much) fewer than $c n^{2}$ crossings. Let us remark that in any rectilinear drawing of $K_{n}$ the edges on the boundary of the convex hull do not cross any other edge. On the other hand, Harborth and Thürmann [8] found a simple topological complete graph in which each edge crosses some other edges.

Let $h=h(n)$ be the smallest integer such that every simple topological complete graph on $n$ vertices contains an edge crossing at most $h$ other edges. Harborth and Thürmann [8] proved $h(n)>\left(\frac{3}{4}+o(1)\right) n$. Other related questions were studied e.g. in [5, 6, 7, 16, 17. It has been asked in the preliminary version of the book [2] whether $h(n)=O(n)$, and the final version of [2] contains a conjecture that $h(n)=o\left(n^{2}\right)$. In this paper we show that $h(n)$ grows much faster and we also give the first subquadratic upper bound on $h(n)$ :

\section{Theorem 1.}

$$
\Omega\left(n^{3 / 2}\right) \leq h(n) \leq O\left(n^{2} / \log ^{1 / 4} n\right)
$$

We describe two essentially different constructions giving the lower bound. We present both of them, since they may help in closing the gap between the bounds given in Theorem 11. We conjecture that the lower bound is closer to the asymptotic behavior of $h(n)$ than the upper bound, and maybe even $h(n)=$ $\Theta\left(n^{3 / 2}\right)$. We remark that our proof gives a reasonable constant involved in the $\Omega$-notation in the lower bound in Theorem 1, For simplicity of presentation, we do not compute the constants. 
It is interesting that for other surfaces (torus, Klein bottle, real projective plane) it is possible to find simple topological complete graphs with each edge crossing $\Omega\left(n^{2}\right)$ other edges. This is discussed in the last section of the paper.

Brass, Moser, and Pach [2] describe a connection between the function $h(n)$ and the maximum number of disjoint edges in a topological graph. They have suggested the following greedy procedure: Select an edge intersecting the smallest number of other edges, delete these edges, and repeat the procedure. The lower bound in Theorem 1 indicates limits of this procedure in some cases. We remark that finding many disjoint edges and various similar questions on topological and geometric graphs have recently received a lot of attention, e.g. see [3, 4, 10, 11, $12,13,14,15$.

\section{The Lower Bound}

\subsection{First Construction}

Let $\mathcal{S}$ be the unit sphere in $\mathbf{R}^{3}$. Our topological complete graph giving the lower bound in Theorem 1 will be drawn on $\mathcal{S}$ by choosing an appropriate set $P_{n}$ of $n$ points on $\mathcal{S}$ and then connecting each pair of points of $P_{n}$ by the shortest arc contained in $\mathcal{S}$. The points of $P_{n}$ will be "well distributed" on $\mathcal{S}$ and in general position, meaning that no two points of $P_{n}$ are antipodal and no three points of $P_{n}$ lie on a common great circle of $\mathcal{S}$.

The crucial requirement on $P_{n}$ is the following condition:

(C) If $d=d\left(P_{n}\right)$ denotes the minimum (Euclidean) distance of a pair of points of $P_{n}$ then for any point $q \in \mathcal{S}$, the $1.1 d$-neighborhood of $q$ contains a point of $P_{n}$.

The set $P_{n}$ is constructed as follows. First, we inductively construct $n$ auxiliary points $a_{1}, \ldots, a_{n}$. Choose a point $a_{1} \in \mathcal{S}$ arbitrarily. Now, let $i \in\{1, \ldots, n-1\}$ and suppose that $a_{1}, \ldots, a_{i}$ have already been selected. Then we choose $a_{i+1}$ as a point on $\mathcal{S}$ maximizing the quantity $\min \left\{\left\|a_{1}-a_{i+1}\right\|,\left\|a_{2}-a_{i+1}\right\|, \ldots, \| a_{i}-\right.$ $\left.a_{i+1} \|\right\}$. Clearly, we can slightly perturb the constructed set $\left\{a_{1}, \ldots, a_{n}\right\}$ so that the perturbed set, $P_{n}$, is in general position and satisfies condition $(C)$.

Observe that $(d=) d\left(P_{n}\right)=\Theta(1 / \sqrt{n})$ follows from the following three facts by a simple counting argument: (i) the area of $\mathcal{S}$ is $\Theta(1)$, (ii) the $1.1 d$-neighborhoods of the points of $P_{n}$ cover $\mathcal{S}$, and (iii) the $0.49 d$-neighborhoods of the points of $P_{n}$ are pairwise disjoint.

Let $T=T_{n}$ be the simple topological complete graph on $\mathcal{S}$ such that $V(T)=$ $P_{n}$ and that $E(T)$ consists of the shortest curves on $\mathcal{S}$ connecting the pairs of vertices. We have to show that every edge in $T$ crosses $\Omega\left(n^{3 / 2}\right)$ other edges.

We use the notions equator, northern/southern hemisphere of $\mathcal{S}$ in the obvious way. Clearly, for any two vertices $a, b$, the edge $a b$ is a portion of the great circle containing $a, b$. Thus, it suffices to show that if a portion $I$ of a great circle of $\mathcal{S}$ has length $|I|=d$ then it is intersected by at least $\Omega\left(n^{3 / 2}\right)$ edges of $T$. We may suppose that $I$ is a portion of the equator. We denote the end-points of $I$ by $s$ 


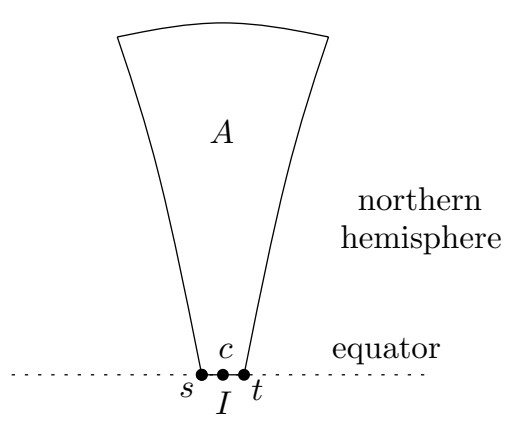

southern hemisphere

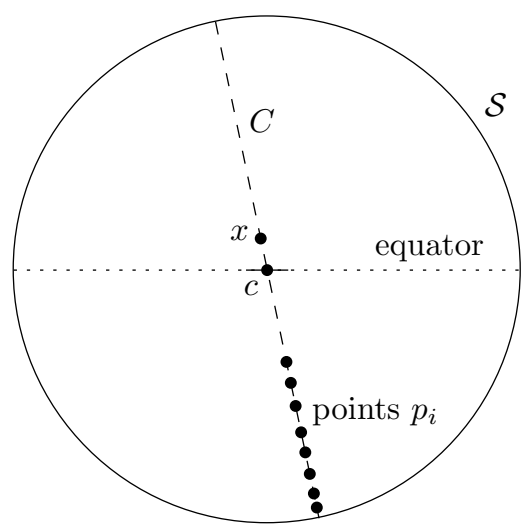

Fig. 1. The region $A$ (left) and the points $p_{i}$ (right)

and $t$. For a point $x \in \mathcal{S}$ not lying on the equator, the spherical triangle stx is the region on $\mathcal{S}$ bounded by $I$ and by the two shortest arcs contained in $\mathcal{S}$ and joining $x$ with the points $s$ and $t$, respectively.

Let $c$ be the mid-point of the $\operatorname{arc} I$. We consider the region $A$ on $\mathcal{S}$ of the points $x$ on the northern hemisphere such that $\|x-c\|<\frac{1}{100}$ and that the spherical triangle $s t x$ has the inner angles at $s$ and $t$ each at most $0.6 \pi$ (see Figure 11). The region $A$ is bounded by $I$ and by three arcs of length $\Theta(1)$. Clearly, its area is $\Theta(1)$ and it contains $\Theta(n)$ points of $P_{n}$. It suffices to show that any point of $A \cap P_{n}$ is an end-point of $\Omega(\sqrt{n})$ edges intersecting $I$.

Let $x \in A \cap P_{n}$. Consider the great circle $C$ going through the points $x$ and $c$ (see Figure 1). Since $d=\Theta(1 / \sqrt{n})$, it is possible to select $\Theta(\sqrt{n})$ points $p_{1}, p_{2}, \ldots, p_{t}$ in the intersection of $C$ with the southern hemisphere such that $\frac{1}{10}<\left\|c-p_{i}\right\|<\sqrt{2}$ (for each $i$ ) and $\left\|p_{i}-p_{j}\right\|>2.2 d$ (for any $i \neq j$ ). In general, the points $p_{i}$ do not lie in $P_{n}$. However, the $1.1 d$-neighborhood of each $p_{i}$ contains a point $p_{i}^{\prime} \in P_{n}$. By the choice of the points $p_{i}$, the points $p_{i}^{\prime}$ are pairwise distinct. It is not difficult to verify that each of the $\Theta(\sqrt{n})$ edges $x p_{i}^{\prime}$ intersects the $\operatorname{arc} I$. This completes the proof that any edge in $T=T_{n}$ crosses $\Omega\left(n^{3 / 2}\right)$ other edges.

\subsection{Second Construction}

Our second construction giving the lower bound in Theorem 1 is only briefly outlined in this extended abstract. We start with any fixed simple topological complete graph $T$ in which each edge has at least one crossing, e.g with the drawing on Fig. 2, Let $V(T)=\left\{v_{1}, v_{2}, \ldots, v_{t}\right\}$. Let $n \geq t$ and suppose for simplicity that $\sqrt{n / t}$ is an integer. We replace each vertex $v_{i}$ by a set $V_{i}$ of $n / t$ vertices placed in a square lattice $\sqrt{n / t} \times \sqrt{n / t}$ of a very small diameter. Any two vertices in distinct sets $V_{i}, V_{j}, i \neq j$, will be connected by an edge contained in a small neighborhood of the edge $v_{i} v_{j}$ of $T$. Let $i \in\{1, \ldots, t\}$ and suppose that the edges in $T$ incident to $v_{i}$ leave the vertex $v_{i}$ in a counterclockwise order 


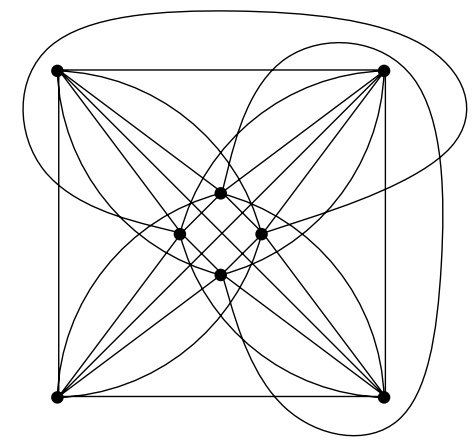

Fig. 2. A simple topological complete graph on 8 vertices in which each edge crosses another edge

$v_{i} v_{j_{1}}, v_{i} v_{j_{2}}, \ldots, v_{i} v_{j_{t-1}}$. In a small neighborhood of the convex hull of $V_{i}$ we draw the edges leaving from the vertices of $V_{i}$ so that any edge connecting a vertex of $V_{i}$ with a vertex of $V_{j_{1}}$ leaves the vertex of $V_{i}$ along a vector parallel to some vector $(1, \varepsilon)$, where $\varepsilon>0$ is very small (and different for different edges), and similarly any edge connecting any vertex of $V_{i}$ with a vertex of $V_{j_{2}}, V_{j_{3}}$, or $V_{j_{4}} \cup \ldots \cup V_{j_{t-1}}$ (respectively) leaves the vertex of $V_{i}$ along a vector parallel to some vector $(\varepsilon, 1)$, $(-1, \varepsilon)$, or $(\varepsilon,-1)$ (respectively). This ensures that after a very tiny perturbation of $V_{i}$ and after connecting any two vertices of $V_{i}$ by a straight-line segment, each such segment (edge) will be intersected by at least $(\sqrt{n / t}-1) n / t=\Theta\left(n^{3 / 2}\right)$ edges connecting vertices of $V_{i}$ with the vertices of $V_{j_{1}} \cup V_{j_{2}} \cup V_{j_{3}} \cup V_{j_{4}}$. It is not too difficult to check that the whole construction can be done so that the resulting drawing is a simple topological (complete) graph. Moreover, any edge connecting vertices from distinct sets $V_{i}, V_{j}$ has $(n / t)^{2}=\Theta\left(n^{2}\right)$ crossings in a small neighborhood of the point where the edge $v_{i} v_{j}$ crosses another edge of the graph $T$. Thus the obtained topological graph gives the lower bound in Theorem 1.

\section{The Upper Bound}

Topological graphs $G, H$ are said to be weakly isomorphic, if there exists an incidence preserving one-to-one correspondence between $(V(G), E(G))$ and $(V(H)$, $E(H))$ such that two edges of $G$ intersect if and only if the corresponding two edges of $H$ do. Let $C_{m}$ denote a complete convex geometric graph with $m$ vertices (note that all such graphs are weakly isomorphic to each other). A simple topological complete graph with $m$ vertices is called twisted and denoted by $T_{m}$, if there exists a canonical ordering of its vertices $v_{1}, v_{2}, \ldots, v_{m}$ such that for every $i<j$ and $k<l$ two edges $v_{i} v_{j}, v_{k} v_{l}$ cross if and only if $i<k<l<j$ or $k<i<j<l$ (see Figure 3). Figure 4 shows an equivalent drawing of $T_{m}$ on the cylindric surface. If $G, H$ are topological graphs, we say that $G$ contains $H$, if $G$ has a topological subgraph weakly isomorphic to $H$. 


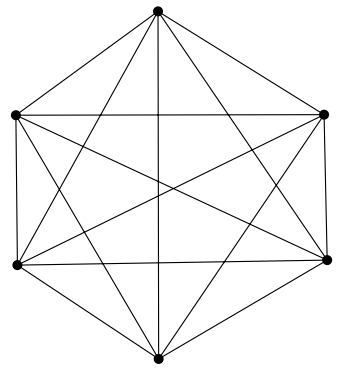

$C_{6}$

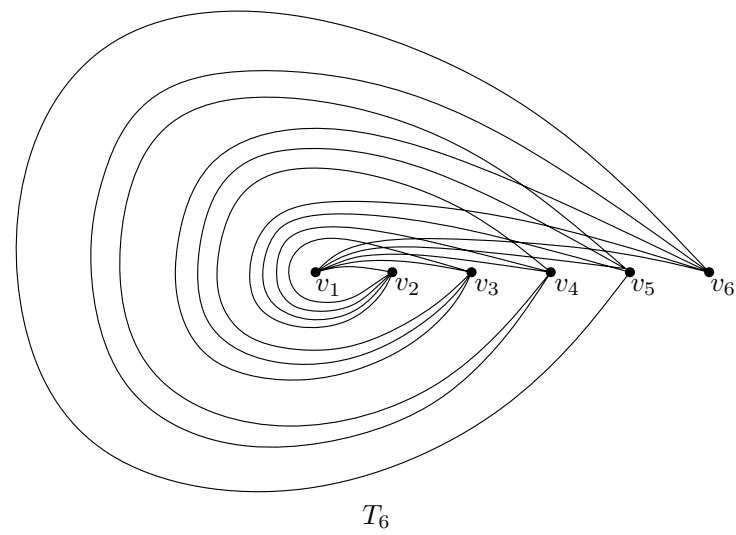

Fig. 3. The convex geometric graph $C_{6}$ and the twisted graph $T_{6}$

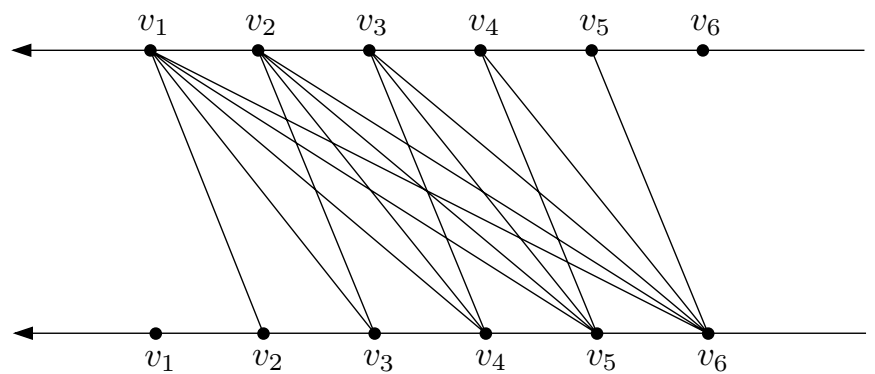

Fig. 4. Drawing of the twisted graph $T_{6}$ on the cylindric surface

In the proof of the upper bound, we will use the following asymmetric form of the result of Pach, Solymosi and Tóth [13]:

Theorem 2. [13] There exists a $c>0$ such that for all positive integers $n, m_{1}$, $m_{2}$ satisfying $m_{1} m_{2} \leq c \log ^{1 / 4} n$ every simple topological complete graph with $n$ vertices contains $C_{m_{1}}$ or $T_{m_{2}}$.

We will use this theorem for $m_{1}=c^{\prime} \log ^{1 / 4} n$ and $m_{2}$ constant.

Now we prove two lemmas, the first one related to the complete geometric graph $C_{m}$, the second one related to the twisted drawing $T_{5}$.

Lemma 1. Let $G$ be a simple topological complete graph with $n$ vertices. If $G$ contains $C_{m}$, then there exists an edge in $G$ which crosses at most $2 n^{2} / m$ other edges.

Proof. Let $H$ be a topological complete subgraph of $G$ with $m$ vertices weakly isomorphic to $C_{m}$. $H$ has a face $F$ that is bounded by a non-crossing Hamiltonian cycle $C$ consisting of $m$ edges. Without loss of generality, suppose that $F$ is the outer face of $H$. Then all edges of $H$ lie inside the region bounded by the cycle $C$. We denote this region by $R$. 
Claim. Let $c$ be a simple continuous curve which starts and ends inside $F$, does not go through any vertex of $H$ and crosses each edge of $H$ at most once. Then $c$ crosses at most two edges of the cycle $C$.

Proof. For contradiction, suppose that $c$ crosses more than two edges of $C$. Then the intersection of $c$ with $R$ consists of $k \geq 2$ disjoint $\operatorname{arcs} c_{1}, c_{2}, \ldots, c_{k}$ (see Figure 5). In the region $R$, the $\operatorname{arcs} c_{1}, c_{2}$ separate two portions of $C$, denoted by $\alpha, \beta$, from each other (see Figure 5 ). Since $c \supseteq c_{1} \cup c_{2}$ intersects each edge of $C$ at most once, each of the arcs $\alpha, \beta$ contains a vertex of $G$. However, any edge $e$ connecting a vertex on $\alpha$ with a vertex on $\beta$ intersects both $c_{1}$ and $c_{2}$. Thus, it intersects $c$ more than once - a contradiction.

Let $c$ be an arbitrary edge of $G$ and let $k$ be the number of edges of $C$ that are crossed by $c$. First, we delete from $c$ a small neighborhood of its end-points, receiving a curve $c^{\prime}$ that is disjoint with all vertices of $H$ and crosses the same edges as $c$ does. If some of the end-points of $c^{\prime}$ lies inside the region $R$, we delete from $c^{\prime}$ the initial part between the end-point and the first point $a$, at which $c^{\prime}$ crosses $C$, including a small neighborhood of $a$. We receive a curve $c^{\prime \prime}$ that has both its end-points inside $F$ and crosses at least $k-2$ edges from $C$. By the previous claim, $c^{\prime \prime}$ crosses at most 2 edges from $C$, thus $k \leq 4$.

$G$ has less than $\frac{n^{2}}{2}$ edges, thus there are at most $2 n^{2}$ crossings between the edges of $G$ and the edges of $C$. By the pigeon-hole principle, among the $m$ edges of $C$ there is an edge, which crosses at most $2 n^{2} / m$ edges of $G$.

Consider a simple topological complete graph $H$ weakly isomorphic to the twisted graph $T_{m}$ with the canonical ordering $v_{1}, v_{2}, \ldots, v_{m}$ of its vertices. The face incident with the vertices $v_{m-1}$ and $v_{m}$ only is called an outer face of $H$ (it coincides with the outer face of the drawing of $T_{m}$ at Figure 3), similarly the face incident with the vertices $v_{1}$ and $v_{2}$ only is called an inner face of $H$.

Lemma 2. Let $H$ be a simple topological complete graph weakly isomorphic to $T_{5}$. There does not exist a simple continuous curve $c$, which crosses each edge of $H$ at most once, does not go through any vertex of $H$, begins and ends inside the outer face of $H$ and intersects the inner face of $H$.

Proof. Let $v_{1}, v_{2}, \ldots, v_{5}$ be the canonical ordering of the vertices of $H$. Consider a Hamiltonian cycle $H_{5}$, which is a subgraph of $H$ with the edge set $E\left(H_{5}\right)=$
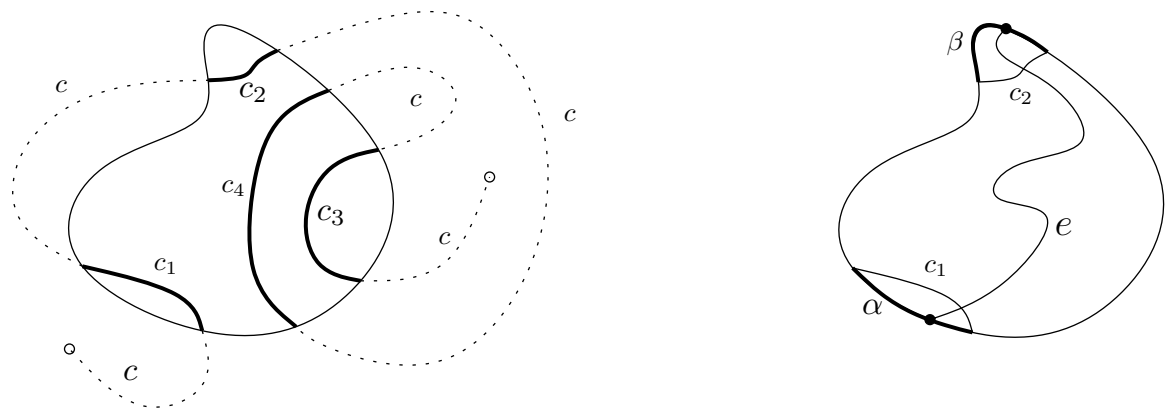

Fig. 5. The $\operatorname{arcs} c_{i}$ (left) and the $\operatorname{arcs} \alpha, \beta$ (right) 
$\left\{v_{1} v_{2}, v_{2} v_{3}, v_{3} v_{4}, v_{4} v_{5}, v_{5} v_{1}\right\}$. Let $F_{1}, F_{2}, F_{3}, F_{4}$ be the four faces of $H_{5}$ such that $F_{1}$ is incident with the vertices $v_{1}$ and $v_{2}$ only and $F_{i}$ borders with $F_{i+1}, i=1,2,3$ (see Figure 6). Note that $F_{1}\left(F_{4}\right)$ is the inner (outer) face of $H$ and that $F_{i}$ does not border with $F_{j}$ if $|i-j| \geq 2$.

For contradiction, suppose that there exists a simple continuous curve $c$ starting and ending inside $F_{4}$ and passing through $F_{1}$, avoiding all vertices of $H$ and crossing each edge of $H$ at most once. Choose a point $p \in c \cap F_{1}$. By the previous observation, between the starting point and $p, c$ has to pass through the faces $F_{2}$ and $F_{3}$, so it must cross at least three edges of $H_{5}$. Similarly, $c$ crosses at least three edges of $H_{5}$ between the point $p$ and its end-point. But $H_{5}$ has only five edges, thus at least one of them is crossed by $c$ more than once, a contradiction.

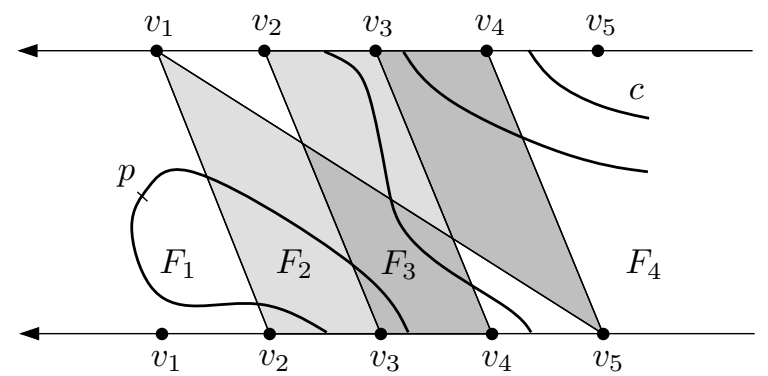

Fig. 6. The graph $H_{5}$ and a curve $c$ with six crossings

The following theorem gives the upper bound in Theorem 1 .

Theorem 3. There exists a $c>0$ such that in every simple topological complete graph with $n$ vertices there exists an edge that crosses at most $\mathrm{cn}^{2} / \log ^{1 / 4} n$ other edges.

Proof. Let $G$ be a simple topological complete graph with $n$ vertices. By Theorem 2, every induced subgraph of $G$ with at least $n^{1 / 8}$ vertices contains $T_{20}$ or $C_{\frac{c^{\prime}}{2} \log ^{1 / 4} n}$. If $G$ contains $C_{\frac{c^{\prime}}{2} \log ^{1 / 4} n}$ then, by Lemma 1, $G$ has an edge which crosses at most $\frac{4}{c^{\prime}} n^{2} / \log ^{1 / 4} n$ other edges. For the rest of the proof, suppose that $G$ does not contain $C_{\frac{c^{\prime}}{2} \log ^{1 / 4} n}$, thus every induced subgraph of $G$ with at least $n^{1 / 8}$ vertices contains $T_{20}$.

Let $T_{20}^{1}$ be a complete subgraph of $G$ with 20 vertices weakly isomorphic to $T_{20}$ and let $v_{1}^{1}, v_{2}^{1}, \ldots, v_{20}^{1}$ be a canonical ordering of its vertices. Consider a graph $H^{1}$ with the vertex set $V\left(H^{1}\right)=V\left(T_{20}^{1}\right)$ and the edge set $E\left(H^{1}\right)=$ $\left\{v_{1}^{1} v_{2}^{1}, v_{2}^{1} v_{3}^{1}, \ldots, v_{19}^{1} v_{20}^{1}, v_{1}^{1} v_{5}^{1}, v_{6}^{1} v_{10}^{1}\right\}$ (see Figure 7). Denote the faces of $H^{1}$ as $F_{1}^{1}, F_{2}^{1}, \ldots, F_{7}^{1}$ such that $F_{1}^{1}$ is the inner face of $T_{20}^{1}, F_{7}^{1}$ contains the outer face of $T_{20}^{1}$ and $F_{i}^{1}$ borders with $F_{i+1}^{1}, i=1,2, \ldots, 6$ (as on the Figure 7).

Applying Lemma 2 on the twisted induced subgraph of $T_{20}^{1}$ with the vertices $v_{1}^{1}, v_{2}^{1}, \ldots, v_{5}^{1}$ we get that every edge of $G$, which crosses $v_{1}^{1} v_{2}^{1}$, has at least one 


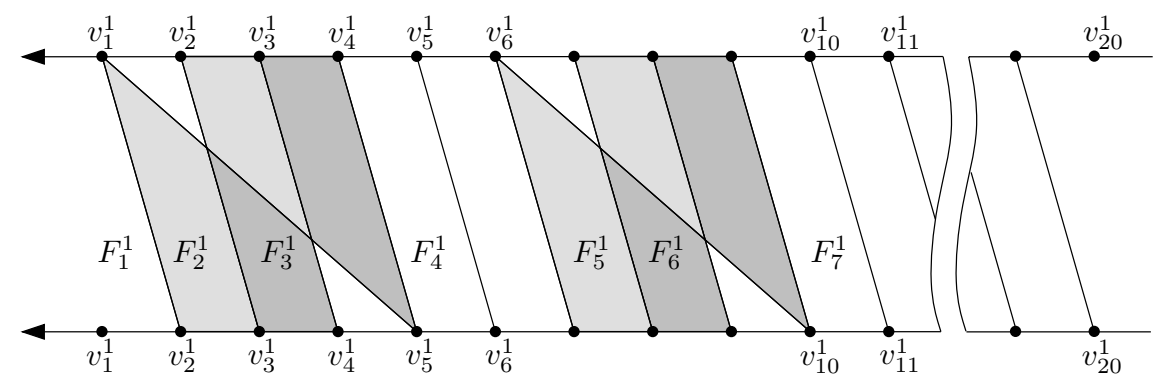

Fig. 7. The graph $H^{1}$ and its seven faces

end-point in the set $A^{1}=F_{1}^{1} \cup F_{2}^{1} \cup F_{3}^{1} \cup\left\{v_{3}^{1}, v_{4}^{1}, v_{5}^{1}\right\}$. Denote $a^{1}=\mid A^{1} \cap$ $V(G) \mid$. If $a^{1}<n^{1 / 8}$, then there are at most $n \cdot n^{1 / 8}=n^{9 / 8}$ edges with one end-point in $A^{1}$, thus at most $n^{9 / 8}$ edges cross the edge $v_{1}^{1} v_{2}^{1}$. In the other case, the complete subgraph of $G$ induced by the set $A^{1} \cap V(G)$ has a subgraph $T_{20}^{2}$ weakly isomorphic to $T_{20}$. Consider a twisted subgraph $H$ of $T_{20}^{1}$ induced by the vertices $v_{10}^{1}, v_{9}^{1}, v_{8}^{1}, v_{7}^{1}, v_{6}^{1}$ (in this canonical ordering). Every edge of $T_{20}^{2}$ has both its end-points inside the outer face of $H$, so it cannot intersect the inner face of $H$ (by Lemma 2). This yields that all edges of $T_{20}^{2}$ lie in the set $B^{1}=F_{1}^{1} \cup F_{2}^{1} \cup \ldots \cup F_{6}^{1} \cup\left\{v_{3}^{1}, v_{4}^{1}, \ldots, v_{10}^{1}\right\}$. Denote $b_{1}=\left|B^{1} \cap V(G)\right|$. Note that $A^{1} \subseteq B^{1}$, thus $a_{1} \leq b_{1}$. It follows that at most one face of the graph $T_{20}^{2}$ does not lie in $B^{1}$. So we can choose a canonical ordering $v_{1}^{2}, v_{2}^{2}, \ldots, v_{20}^{2}$ of the vertices of $T_{20}^{2}$ such that the faces $F_{1}^{2}, F_{2}^{2}, \ldots, F_{6}^{2}$ of the graph $H^{2}$ (defined analogically as $H^{1}$ and its faces $F_{i}^{1}$ ) lie in $B^{1}$. We define sets $A^{2}, B^{2}$ and numbers $a^{2}, b^{2}$ analogically as $A^{1}, B^{1}, a^{1}, b^{1}$. $B^{2}$ is a proper subset of $B^{1}$, since all vertices of $T_{20}^{2}$ and faces $F_{1}^{2}, F_{2}^{2}, \ldots, F_{6}^{2}$ are contained in $B^{1}$, but, for example, vertex $v_{11}^{2}$ does not lie in $B^{2}$. It yields that $b_{2}<b_{1}$. If $a_{2} \geq n^{1 / 8}$, then there exists a twisted complete subgraph $T_{20}^{3}$ of $G$ induced by some 20 vertices of the set $A^{2} \cap V(G)$. Further we proceed by induction, similarly as above. In the $i$-th step, assuming that $a_{i-1} \geq n^{1 / 8}$, we find a twisted complete subgraph $T_{20}^{i}$ of $G$ with 20 vertices and define two integers $a_{i}, b_{i}$ satisfying $0 \leq a_{i} \leq b_{i}<b_{i-i}$. After finitely many steps, we get a number $a_{i}$, which is less than $n^{1 / 8}$. It means that the edge $v_{1}^{i} v_{2}^{i}$ in the graph $T_{20}^{i}$ is crossed by less than $n^{9 / 8}<n^{2} / \log ^{1 / 4} n$ other edges of $G$.

\section{Other Surfaces}

Here we show that an analogue of the function $h(n)$ is quadratic for the torus and for the Klein bottle1:

\footnotetext{
${ }^{1}$ The same result for the projective plane has been recently found by Attila Pór (personal communication). We describe Pór's construction at the end of this section. Since any drawing of a finite graph on the projective plane can be easily transformed to a drawing on the Klein bottle, Pór's construction can be used to obtain an alternative proof of Proposition 1 for the Klein bottle.
} 
Proposition 1. On the torus and on the Klein bottle, there exists a simple topological complete graph with each edge having at least $\mathrm{cn}^{2}$ crossings.

Proof. Consider a rectangle from which, after gluing its opposite sides, we get a torus. Place the vertices $v_{1}, v_{2}, \ldots v_{n}$ along its upper and lower side in this order. We draw the edges the following way: if $j-i \bmod n \leq\left\lfloor\frac{n-1}{2}\right\rfloor$, or if $j-i \bmod n=\frac{n}{2}$ and $i \leq \frac{n}{2}$, we represent the edge $v_{i} v_{j}$ as a segment starting at the upper vertex $v_{i}$, directing down and to the right, possibly leaving the rectangle on the right-hand side and entering on the left-hand side and ending at the lower vertex $v_{j}$. At Figure 8 , you can see the representation of the edges incident to one vertex. It is clear that in this drawing each two edges intersect at most once and that every edge crosses at least $c n^{2}$ other edges.

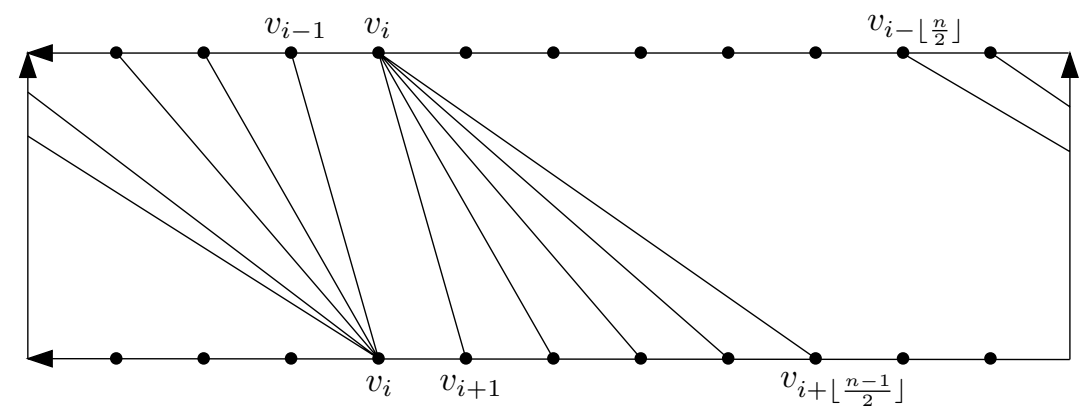

Fig. 8. Edges incident to the vertex $v_{i}$ in the drawing of $K_{n}$ on the torus

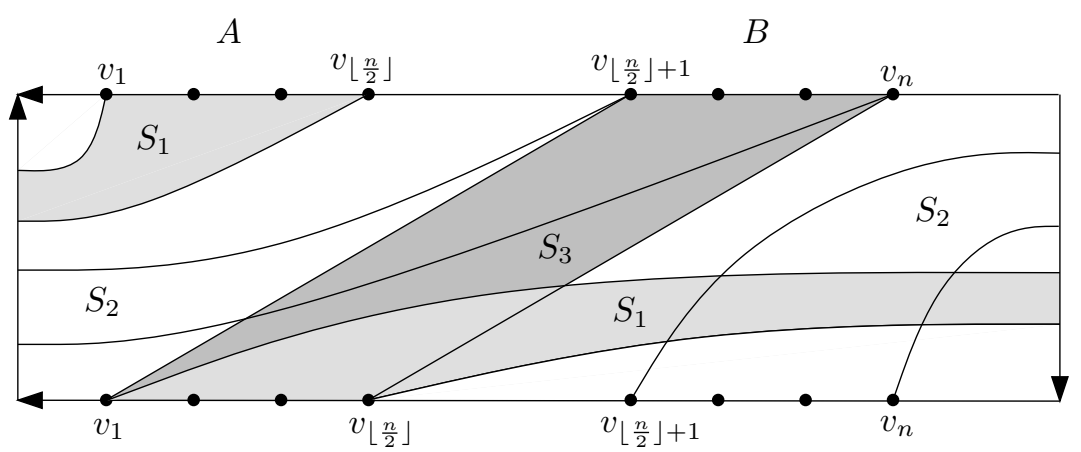

Fig. 9. Drawing of $K_{n}$ on the Klein bottle

For the drawing on the Klein bottle, divide the vertices into two sets $A=$ $\left\{v_{1}, v_{2}, \ldots, v_{\left\lfloor\frac{n}{2}\right\rfloor}\right\}$ and $B=\left\{v_{\left\lfloor\frac{n}{2}\right\rfloor+1}, \ldots, v_{n}\right\}$ and place all the edges into three strips $S_{1}, S_{2}, S_{3}$. $S_{1}$ contains all edges among the vertices of $A, S_{2}$ contains all edges among the vertices of $B$, and $S_{3}$ all edges between $A$ and $B$ (see Figure 9). Clearly, we can draw the edges such that no two of them intersect more than once. It is not difficult to verify that each edge crosses at least $c n^{2}$ other edges. 
We now describe Attila Pór's construction of a simple topological complete graph on $n$ vertices with each edge intersecting at least $\Omega\left(n^{2}\right)$ other edges. The projective plane can be obtained by adding a line at infinity to the real plane. We place the vertices of the constructed topological graph in the vertices of a regular $n$-gon $P$. Any two vertices are connected by the portion of the line through the two vertices outside of the polygon $P$. It is easy to see that any edge is intersected by $\Omega\left(n^{2}\right)$ other edges.

\section{References}

1. M. Ajtai, V. Chvátal, M. Newborn, and E. Szemerédi: Crossing-free subgraphs. Annals of Discrete Mathematics 12 (1982), 9-12

2. P. Brass, W. Moser, and J. Pach: Research problems in discrete geometry. Springer (2005)

3. G. Cairns and Y. Nikolayevsky: Bounds for generalized thrackles. Discrete Comput. Geom. 23 (2000), 191-206

4. J. Černý: Geometric graphs with no three disjoint edges. Discrete Comput. Geom. (to appear)

5. H. Harborth: Crossings on edges in drawings of complete multipartite graphs. Colloquia Math. Soc. János Bolyai 18 (1978), 539-551

6. H. Harborth and M. Mengersen: Edges without crossings in drawings of complete graphs. J. Comb. Theory, Ser. B 17 (1974) 299-311

7. H. Harborth and M. Mengersen: Drawings of the complete graph with maximum number of crossings. Congr. Numerantium 88 (1992), 225-228

8. H. Harborth and C. Thürmann: Minimum number of edges with at most $s$ crossings in drawings of the complete graph. Congr. Numerantium 102 (1994), 83-90

9. F.T. Leighton: New lower bound techniques for VLSI. Math. Systems Theory 17 (1984), 47-70

10. J. Pach, R. Pinchasi, G. Tardos, and G. Tóth: Geometric graphs with no selfintersecting path of length three. Graph drawing 2002, 295-311, Lecture Notes in Comput. Sci., 2528, Springer, Berlin, 2002; also European J. Combin. 25 (2004), no. $6,793-811$

11. J. Pach, R Radoičić, and G. Tóth: A generalization of quasi-planarity. Towards a theory of geometric graphs, 177-183, Contemp. Math., 342, Amer. Math. Soc., Providence, RI, 2004

12. J. Pach, R Radoičić, and G. Tóth: Relaxing planarity for topological graphs. Discrete and computational geometry, 221-232, Lecture Notes in Comput. Sci. 2866, Springer, Berlin, 2003

13. J. Pach, J. Solymosi and G. Tóth: Unavoidable configurations in topological complete graphs. Discrete Comput. Geom. 30 (2003), 311 - 320

14. J. Pach and G. Tóth: Disjoint edges in topological graphs. To appear

15. R. Pinchasi and R Radoičić: Topological graphs with no self-intersecting cycle of length 4. Towards a theory of geometric graphs, 233-243, Contemp. Math., 342, Amer. Math. Soc., Providence, RI, 2004

16. G. Ringel: Extremal problems in the theory of graphs. Theory Graphs Appl., Proc. Symp. Smolenice 1963, 85-90 (1964)

17. R.D. Ringeisen, S.K. Stueckle, and B.L. Piazza: Subgraphs and bounds on maximum crossings. Bull. Inst. Comb. Appl. 2 (1991), 33-46 